\title{
Nogo-B Promotes Angiogenesis in Proliferative Diabetic Retinopathy via VEGF/PI3K/Akt Pathway in an Autocrine Manner
}

\author{
Yuelu Zhang ${ }^{a}$ Liang Wang ${ }^{b}$ Yuechan Zhang ${ }^{c}$ Mo Wang ${ }^{a}$ Qinglei Sun ${ }^{a}$ \\ Fangzhou Xia ${ }^{a}$ Ruobing Wanga ${ }^{a}$ Lin Liu $^{a}$ \\ aDepartment of Ophthalmology, Renji Hospital, School of Medicine, Shanghai Jiaotong University, \\ Shanghai, 'bepartment of Burn Surgery, Changhai Hospital, Second Military Medical University, \\ Shanghai, 'Department of Pharmacy, Zhangjiagang Hospital of Traditional Chinese Medicine, Nanjing \\ University of Chinese Medicine, Suzhou, China
}

\section{Key Words}

Nogo-B • Angiogenesis • Proliferative diabetic retinopathy

\begin{abstract}
Background/Aims: Nogo- $B$, a conservative protein of endoplasmic reticulum, is a member of the reticulon family of proteins. Proliferative diabetic retinopathy (PDR) is the major concerning problem of diabetic retinopathy. This study explored the role of Nogo-B in the regulation of angiogenesis in PDR patients and primary human retinal endothelial cells (HRMECs). Methods: Nogo-B was down-regulated through the use of Lentivirus-NogoBRNAi, the effects of Nogo-B on angiogenesis under high glucose stimulation were evaluated via CCK-8 assay, wound closure assay, transwell assay, and tube formation assay. Expression of Nogo-B, VEGF, PI3K and Akt were determined by western blotting, immunofluorescence, enzyme-linked immunosorbent assay (ELISA). Co-culture systerm was used to explore cell communication. Results: Nogo-B was highly enriched in ocular tissues of PDR patients and in HRMECs exposed to high glucose. Down-regulation of Nogo-B attenuated high glucose induced cell migration and tube formation in HRMECs. Mechanistically, in comparison with the negative control group, Lentivirus-NogoB-RNAi group had exhibited reduced VEGF secretion, weakened PI3K and Akt activation. Besides, high glucose treatment promoted the secretion of Nogo-B and presented as a "long-term memory". Conclusions: These data collectively indicated that Nogo-B promoted angiogenesis in HRMECs via VEGF/PI3K/Akt pathway in an autocrine manner.
\end{abstract}

Ruobing Wang and Lin Liu
Department of Ophthalmology, Renji Hospital, School of Medicine, Shanghai Jiaotong University, No.160 Pujian Road, Shanghai (China)

Tel. +86-021-25072820, E-Mail liulin1963rj@126.com and adawrb@126.com

\section{KARGER}




\section{Cellular Physiology Cell Physiol Biochem 2017;43:1742-1754

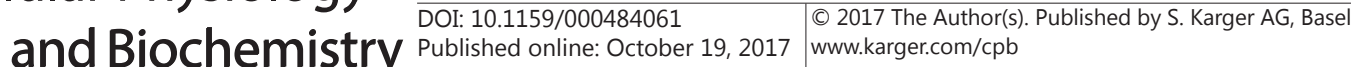 \\ Zhang et al.: Nogo-B Promotes Angiogenesis Via VEGF/PI3K/Akt Pathway}

\section{Introduction}

Diabetes mellitus is one of the most common metabolic diseases and is associated with long-term damage of various organs $[1,2]$. Despite the well-developed diagnosis and medical care, the global prevalence of diabetes will increase to 366 million by 2030 estimated by the World Health Organization [3]. Diabetic retinopathy (DR) is a severe complication of diabetes and remains one of the leading cause of blindness among working-age adults worldwide [4]. The major vision-threatening form of DR is proliferative diabetic retinopathy (PDR), the advanced stage of DR with characteristics of neovascularization [5, 6]. Population-based studies reported an annual incidence PDR rates of $1.5 \%$ in participants of type 1 diabetes and 4-year incidence PDR rates of 2-7\% in those of type 2 diabetes [7-9].

At present, the standard management of PDR is laser photocoagulation and vitreoretinal surgery, nevertheless, both of the treatments are focused on the advanced stage of DR and carry potential side effects $[5,10]$. Hence a wide range of new approaches for DR are investigated including anti-inflammatory agents, G-protein coupled receptor agonists and antagonists, neuroprotectants [4, 11-14]. However, most of the clinical trials of new agents to inhibit progression of DR have been far more disappointing $[3,15]$. Thus, further studies to explore novel pharmacological targets to manage DR are still pressing needed.

The reticulon (RTN) family, which have been identified in most eukaryotic cells, is a transmembrane protein sharing the common $\mathrm{C}$-terminal region encodes the reticulonhomology domain [16]. There are four reticulon genes (RTN1, RTN2, RTN3, RTN4) in mammalian, among which RTN4 (also known as Nogo) has recently been the focus of intensive investigation [16]. Nogo-A, Nogo-B, Nogo-C are the major protein isoforms translated from RTN4 gene, and they share an identical C-terminal domain (Nogo-66) [17]. Despite Nogo-A and Nogo-C are found in the central nervous system, with Nogo-C being additionally detected in skeletal muscle, Nogo-B is widely expressed in most tissues $[18,19]$. Much known is about Nogo-A because of its function as neurite outgrowth inhibitor while the other two isoforms remains largely elusive [20]. This study is concentrated on the second homologues Nogo-B. Previous studies reported that Nogo-B was associated with vascular remodeling upon vascular injury [21]. More recently, it was demonstrated that Nogo-B regulated sphingolipid homeostasis to control blood pressure $[22,23]$. However, little attention was paid to the function of Nogo-B in diabetic vascular pathology especially in PDR.

In the current study, Nogo-B was found to be highly enriched in ocular tissues of PDR patients and in primary human retinal endothelial cells (HRMECs) treated with high glucose. To explore the role of Nogo-B in angiogenesis in PDR and evaluate its potential function as a therapeutic target for the treatment of PDR, we downregulated the expression of Nogo-B using lentivirus in HRMECs, and multiple assays associated with angiogenesis, as well as the expressions of related genes were investigated.

\section{Materials and Methods}

\section{Cell culture}

Primary HRMECs were purchased from Angio-Proteomie (Oxfordshire, UK) and cultured in endothelial cell medium supplemented with 5\% fetal bovine serum (ScienCell, Carlsbad, CA). Cells from passage 3 to 10 were used and incubated with normal D-glucose $(5.5 \mathrm{mM})$, high D-glucose $(25 \mathrm{mM})$ and L-glucose $(25 \mathrm{mM}$, as osmotic control).

\section{Human surgical samples}

All procedures in this study were approved by the Ethics Committee of the Renji Hospital of Shanghai Jiaotong University School of Medicine and adhered to the tenets of the Declaration of Helsinki. All experiments were performed in accordance with relevant guidelines. Vitreous fluids (500-1300 mL) were collected from 17 eyes of 17 patients who had undergone vitrectomy. Among which, non-diabetic macular hole (MH) and rhegmatogenous retinal detachment (RRD) patients were served as control group $\left(\mathrm{n}_{\mathrm{MH}}=5, \mathrm{n}_{\mathrm{RRD}}=5, \mathrm{n}_{\mathrm{PDR}}=\right.$ 


\section{Cellular Physiology Cell Physiol Biochem 2017;43:1742-1754

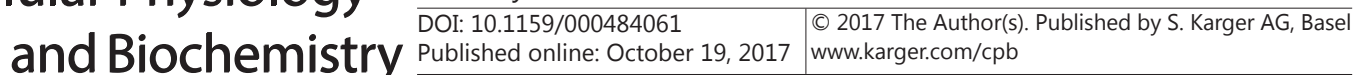 \\ Zhang et al.: Nogo-B Promotes Angiogenesis Via VEGF/PI3K/Akt Pathway}

7). Samples with obvious bleeding were excluded. Vitreous samples were immediately centrifuged at 15 , $000 \mathrm{~g}$ for $20 \mathrm{~min}$ at $4^{\circ} \mathrm{C}$ and the supernatants were stored at $-80^{\circ} \mathrm{C}$ until use. Six fibrovascular samples were surgically removed from the PDR eyes and were used in immunohistochemistry and Immunofluorescence.

\section{Immunohistochemistry}

Fibrovascular samples were fixed in $4 \%$ paraformaldehyde and embedded in paraffin. The fixed sections were stained with Nogo-B antibody (abcam, Cambridge, USA). Nuclei were counterstained with hematoxylin and sections were visualized with light microscopy.

\section{ELISA assay for Nogo-B and VEGF}

Concentrations of Nogo-B in vitreous humor was measured using human soluble Nogo-B elisa kit (Biolegend, San Diego, CA) according to the manufacturer's instruction as previously described [24]. Concentrations of in the cell supernatant was measured by human VEGF ELISA kit (R\&D, Minneapolis, USA). Assays were performed in three replicated wells and each experiment was repeated three times independently.

\section{Immunofluorescence}

Paraffin sections of fibrovascular samples were deparaffinised and hydrated by exposure to xylene and graded alcohols followed by water. And microwave-based antigen retrieval was performed in $10 \mathrm{mmol} / \mathrm{l}$ citrate buffer $(\mathrm{pH}=6)$. Then sections were probed with Nogo-B (abcam, Cambridge, USA) and CD31 (abcam, Cambridge, USA). The secondary antibodies used for fluorescence detection were Alexa Fluor 488 and 555 (Invitrogen, Carlsbad, USA). Sections were visualised under microscopy.

\section{Lentiviral vector transduction}

Both Lenti-NogoB-RNAi and Lenti-NC were constructed and producted by Genechem (Shanghai, China). $2 \times 10^{5}$ HRMECs were plated in each well of a 6-well-plate. When the cells reached $20 \%$ to $30 \%$ confluence, lentiviral vector expressing siRNA-NogoB (5'-3': CACAGAAUCUAUGGACUGAAU) and negative control (5'-3': UUCUCCGAACGUGUCACGUTT) was added (MOI=10) with $50 \mu \mathrm{g} / \mathrm{mL}$ Polybrene (Genechem, Shanghai, China). After incubation for 8 hours, the media replaced with complete culture media. Transduced cell clones were selected with $3 \mu \mathrm{g} / \mathrm{mL}$ puromycin (Genechem, Shanghai, China) for 2 weeks, and cells were expanded in complete culture medium.

\section{CCK-8 assay}

HMERCs $\left(2 \times 10^{4}\right.$ cells/well) were seeded into 96-well plates. After attachment, cells were incubated with different glucose for 48 hours. Cell viability was determined by CCK-8 (Dojindo, Rockville, USA). The optical density values at $450 \mathrm{~nm}$ were quantified by a microplate reader (BioTek, Winooski, USA). Assays were performed in three replicated wells and each experiment was repeated three times independently.

\section{Wound closure assay}

Confluent monolayers of HRMECs were wounded using a micropipette tip (200 $\mu \mathrm{L}$ tip), rinsed with PBS to remove detached cells, and incubated with cell culture medium without fetal bovine serum. Wound closure was monitored after 24 hours and photographed under inverted microscope.

\section{Cell migration assay}

HRMECs $\left(1 \times 10^{5}\right.$ cells/well $)$ were seeded into the upper chamber of the transwells, and then high D-glucose $(25 \mathrm{mM})$ or L-glucose $(25 \mathrm{mM})$ was added into the lower chamber. After 12 hours, the nonmigrated cells were wiped off with a cotton swab, and the filters were fixed with $4 \%$ paraformaldehyde for 20 minutes before stained by crystal violet (Beyotime, Shanghai, China). Images were pictured under the inverted microscope.

\section{Tube formation assay}

HRMECs $\left(1 \times 10^{5}\right.$ cells/well $)$ were starved in endothelial cell medium containing $0.1 \%$ fetal bovine serum for 8 hours, and then incubated with different glucose for 48 hours. Cells were seeded into 24-well plates precoated with matrigel (BD Biosciences, Maryland, USA), and treated in different glucose for another 6 hours. Images were pictured under the inverted microscope. 


\section{Cellular Physiology Cell Physiol Biochem 2017;43:1742-1754

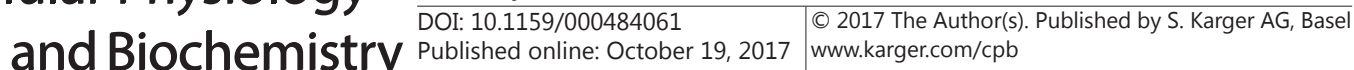

\section{Western-blot analysis}

Protein samples were separated by SDS-PAGE and blots were probed with an appropriate combination of primary and secondary antibodies. The bands were identified by enhanced chemiluminescence kits and visualized using the Amersham Image 600 System. Primary antibodies were antibodies to Nogo-B (Abcam, Cambridge, USA), PI3K p85(Cell Signaling Technology, Danvers, USA), PI3K p-p85 (Cell Signaling Technology, Danvers,USA), Akt (Cell Signaling Technology, Danvers,USA), p-Akt (Cell Signaling Technology, Danvers,USA), GAPDH (Santa Cruz,Dallas,USA). All the second antibodies were obtained from Calbiochem.

\section{Co-culture system}

First, HRMECs infected by lentivirus were cultured on transwell inserts (NC and LV group), and D-glucose $(25 \mathrm{mM})$ was added to both of the inserts. After 48 hour treatment, the cell supernants was collected, and fresh complete growth medium with normal D-glucose was added for further culture. Then normal HRMECs were seeded to the bottom wells of chamber for addtional 24 hours to test their ability of angiogenesis and both the supernants and cells were collected for subsequently examination (symboled NC-f and LV-f group).

\section{Statistical analysis}

Statistical analyses were performed using the SPSS version 20.0. Data were expressed as means \pm standard error of the mean or median with interquattile range. The significance of differences between groups was evaluated by two-tailed student's t-test, non-parametric tests and one-way ANOVA test, and $P<$ 0.05 was considered as statistically significant differences.

\section{Results}

Nogo-B was highly expressed in human PDR tissues

Since no studies on Nogo-B expression in human ocular tissue have been reported, first, using assays of immunohistochemisty, we confirmed the expression of Nogo-B in fibrovascular tissues surgically excised from human eyes (Fig. 1a). Next, ELISA analysis on vitreous humor suggested that the expression of Nogo-B in PDR patients was considerably higher than $\mathrm{MH}$ and RRD patients (Fig. 1b). Further, doublestaining experiments of fibrovascular tissue samples demonstrated colocalization of Nogo-B signal with CD31, a vascular endothelial cell marker, showing that Nogo-B protein could be produced by vascular endothelial cells (Fig. 1c).

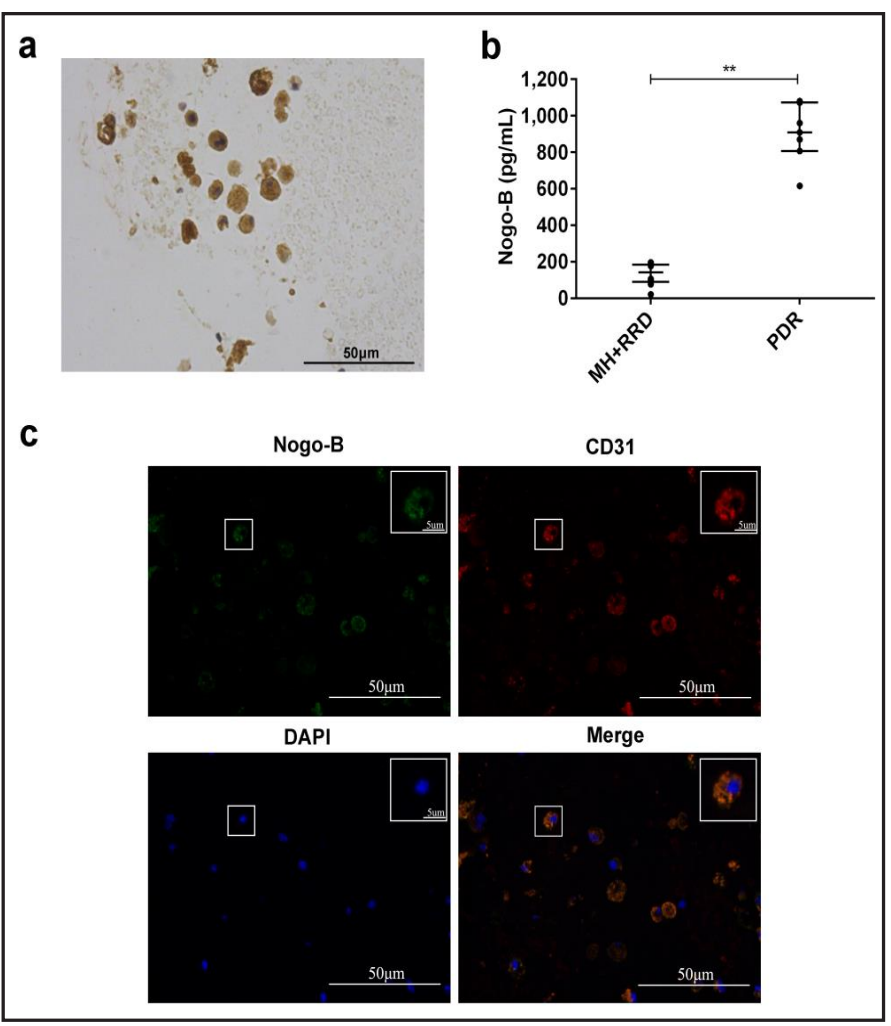

Fig. 1. Nogo-B was highly expressed in human PDR tissues. (a) Nogo-B immunohistochemistry in fibrovascular membranes of patients with PDR, the scale bar was $50 \mu \mathrm{m}$ (b) ELISA tests of Nogo-B in human vitrous fluid samples, the data were represented as median with interquattile range, $\mathrm{n}_{\mathrm{MH}}=5, \mathrm{n}_{\mathrm{RRD}}=5, \mathrm{n}_{\mathrm{PDR}}=7$. ${ }^{* *}, \mathrm{P}<0.01$ by Mann-Whitney $\mathrm{U}$ test (c) Triple labelling of Nogo-B (green), CD31 (red) and DAPI (blue) in the fibrovascular tissues of patients with PDR. The boxed area indicated higher power magnifications. Scale bar was $50 \mu \mathrm{m}$. 
a
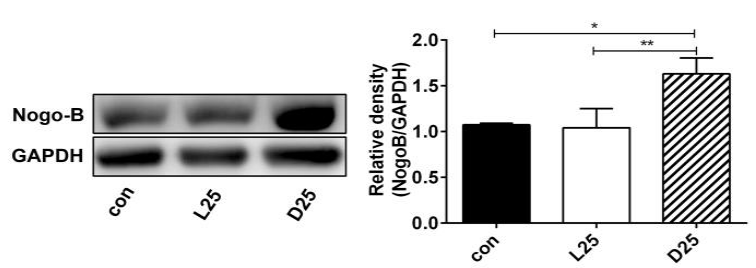

c

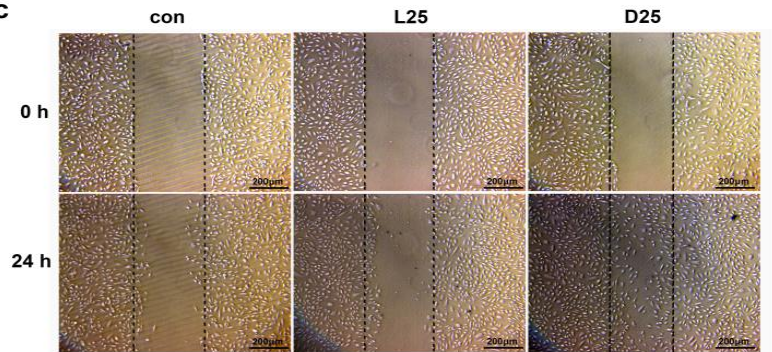

d
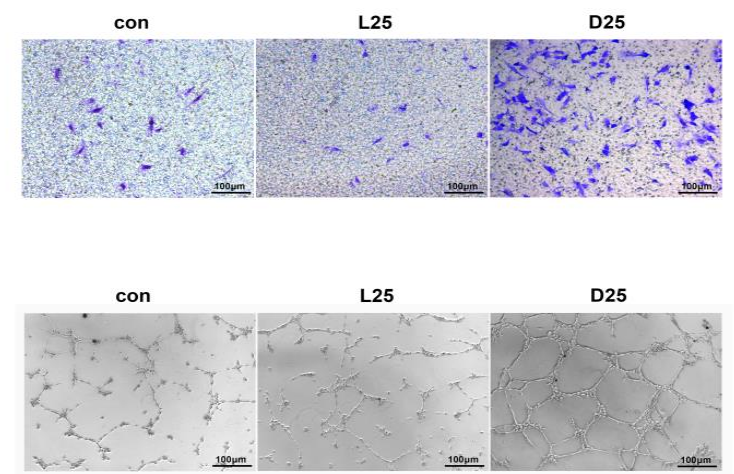

b
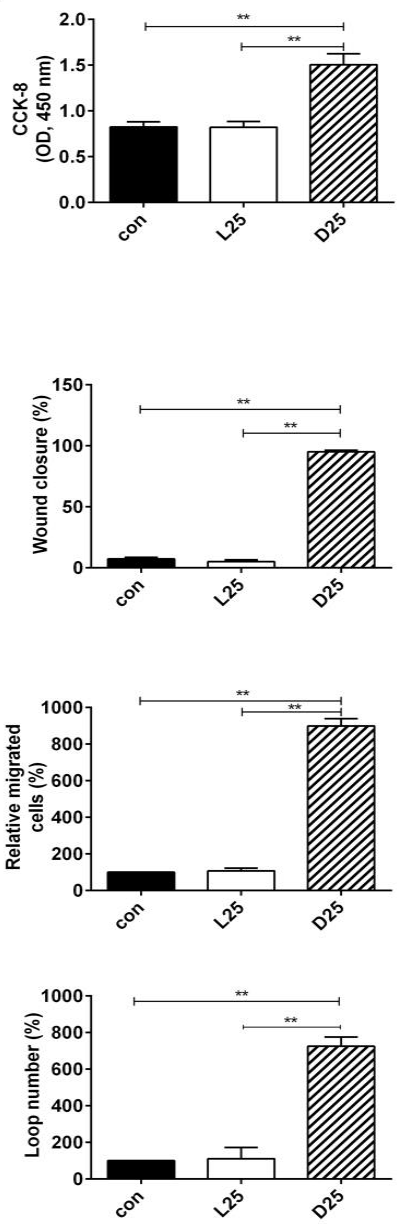

Fig. 2. High glucose induced Nogo-B expression and angiogenesis in HRMECs. Left panel: Nogo-B protein expression in HRMECs exposed to different glucose for 48 hours. Right panel: Representative blots for Nogo-B, $n=3$ in each group (a). The cell viability was quantified and presented as a histogram by CCK-8 assay in HRMECs exposed to different glucose for 48 hours, $n=3$ in each group (b). The migration rate of HRMECs, cultured under various glucose conditions, was determined in scratch wound assay, $\mathrm{n}=3$ in each group (c) and transwell assay, $n=3$ in each group (d). The tube formation ability was assesed by plating the cells on matrigel, $\mathrm{n}=3$ in each group (e). Quantitative assessment of the data was presented in the right. Data were shown as mean $\pm \mathrm{SEM},{ }^{*}, \mathrm{P}<0.05,{ }^{* *}, \mathrm{P}<0.01$ by Tukey HSD test.

High glucose induced Nogo-B expression and angiogenesis in HRMECs.

Angiogenesis, a critical hallmark of pathological changes in PDR patients, is a complicated process involves the proliferation, migration and tubulogenesis of endothelial cells $[25,26]$. In this study, treatment of high D-glucose $(25 \mathrm{mM})$ considerably promotes Nogo-B protein expression compared with osmotic control L-glucose $(25 \mathrm{mM})$ and normal D-glucose control (5.5 mM) (Fig. 2a). To verify whether D-glucose induces angiogenesis, multiple assays were applied in HRMECs. Exposure to D-glucose $(25 \mathrm{mM})$ resulted in markedly enhancement of proliferation in HRMECs as indicated from CCK-8 assay (Fig. 2b). To detected the migration of HRMECs, scar assay and transwell assay were examined. High D-glucose exhibited faster wound closure rate than L-glucose and normal D-glucose control, which is consistant with the transwell results (Fig. 2c, d). Tube formation assay was used to further investigate potential effects of high glucose on tubulogenesis, another critical step responsible for angiogenesis. Formation of tubular-like network was more profound in the presence of high D-glucose, 


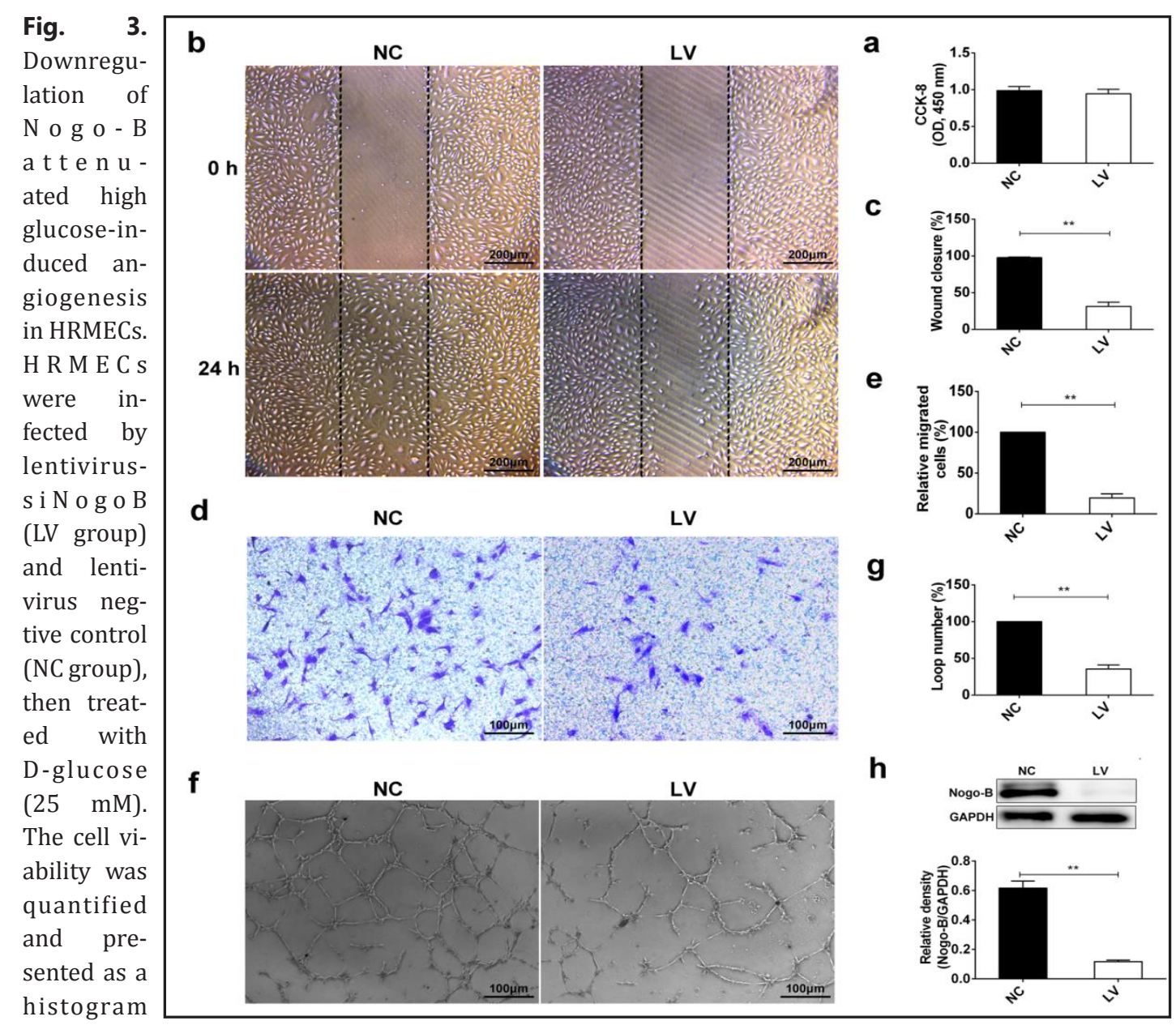

by CCK-8

assay after treatment with D-glucose (25 mM) for 48 hours, $n=3$ in each group (a). The migration rate was determined in scratch wound assay, $n=3$ in each group (b, c) and transwell assay, $n=3$ in each group (d, e). The tube formation ability was assesed by plating the cells on matrigel, $n=3$ in each group (f, g). The expression of Nogo-B infected by lentivirus was detected by western blot (h). Data were shown as mean \pm SEM, ${ }^{* *}$, $\mathrm{P}<0.01$ by student's t-test.

with a few tubes of disconnected networks observed in L-glucose and normal D-glucose control (Fig. 2e).

Downregulation of Nogo-B attenuated high glucose-induced angiogenesis in HRMECs

As high D-glucose promoted Nogo-B expression, we studied the effects of Nogo-B in high glucose-induced angiogenesis using lentivirus downregulating Nogo-B (LV-siNogoB) in HRMECs. Compared with negative control of lentivirus (NC group), downregulating of Nogo-B partially reversed high D-glucose $(25 \mathrm{mM})$ induction of migration and tube formation in HRMECs as indicated from scar assays, transwell assay and tube formation assay(Fig. 3bg). However, downregulation of Nogo-B didn't affect the proliferation rate of HRMECs from CCK-8 assay (Fig. 3a).

Downregulation of Nogo-B blocked high glucose-induced activation of VEGF/PI3K/Akt pathway in HRMECS

Previous studies revealed that activation of the PI3K/Akt pathway was crucial for VEGF induced angiogenesis, including cell survival, migration and tube formation [27-30]. To elucidate the underlying mechanisms for Nogo-B to affect angiogenisis of HRMECs, we 
Fig. 4. Downregulation of Nogo-B blocked high glucose-induced activation of VEGF/PI3K/ Akt pathway in HRMECs. (a) Protein concentrations of VEGF in the cell supernatant were measured by ELISA. Left panel: HRMECs were exposed to different glucose for 48 hours. Right panel: Lentivirus infected HRMECs were exposed to high D-glucose (25 mM) for 48 hours. $\mathrm{n}=3$ in each group. (b) Akt and PI3K p85

was activated after treatment with high D-glucose (25 mM) for 48 hours by using wetern blot, $n=3$ in each group. Quantitative assessment of the data was presented in the right. (c) The glucose-induced activation of PI3K p85 and Akt was blunted after downregulartion of Nogo-B, $n=6$ in each group. Quantitative assessment of the data was presented in the right. Data were shown as mean $\pm \mathrm{SEM},{ }^{* *}, \mathrm{P}<0.01$ by student's t-test.

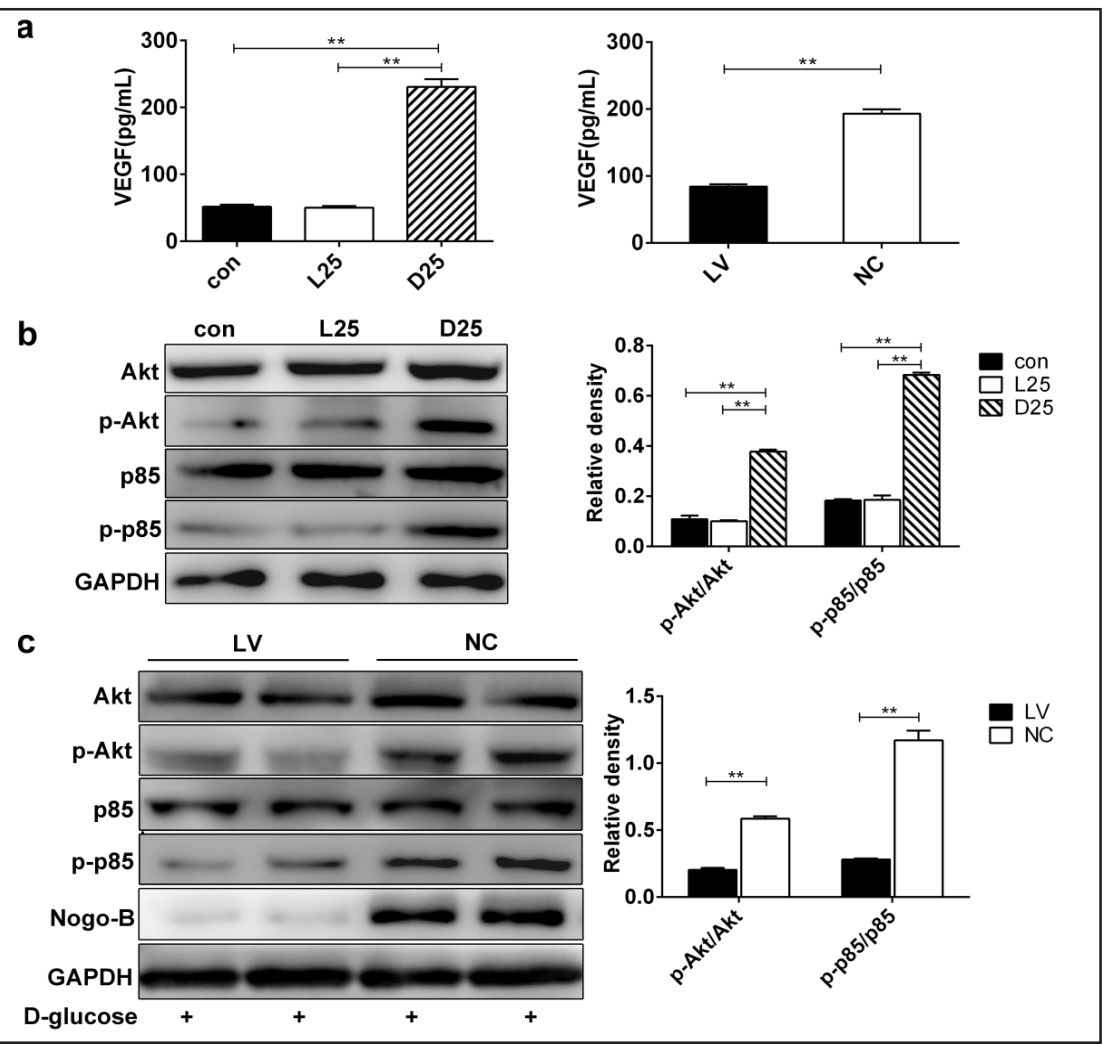

assessed VEGF secretion in lentivirus affected HRMECs cultured in high D-glucose (25 mM). As evidenced by Fig. 4a, high D-glucose (25 mM) treatment stimulated VEGF secretion in the supernatant to a great extent, which was blunted after down-regulation of Nogo-B; Further, high D-glucose was shown to activate PI3K p85 and Akt, which was blocked by down-regulation of Nogo-B, demonstrated that the action of Nogo-B in angiogenesis could correlated with VEGF/PI3K/Akt pathway (Fig. 4b, c).

Recently Nogo-B was shown to inhibit the sphingolipid de novo biosynthesis [22]. Ceramide, a central metabolite of this pathway, has been shown to decrease the activation of the Akt pathway [31]. So we used myriocin to inhibit sphingolipid biosynthesis in Nogo-B knock down HRMECs. It was shown that the rate of migration and tube formation, as well as Akt activation in LV group was increased after myriocin pretreatment (Fig. 5).

High glucose treatment promoted the secretion of Nogo-B and presented as a "long-term memory"

Since western blotting of cell extracts from high D-glucose treatment indicated the elevated expression of Nogo-B, we next tested whether high glucose induced upregulation of Nogo-B could be released. ELISA analysis of cell supernants reflected that HRMECs exposed to high D-glucose for 48 hours induced notable secretion of Nogo-B, compared with L-glucose groups and normal D-glucose control (Fig. 6a). Interestingly, after 48 hour treatment of high D-glucose followed by fresh complete growth medium with normal D-glucose of additional 24 hours, Nogo-B was still apparently detectable and was much more sereted in the high D-glucose group (Fig. 6b). Next, we tested whether this phenomenon could be observed 
a
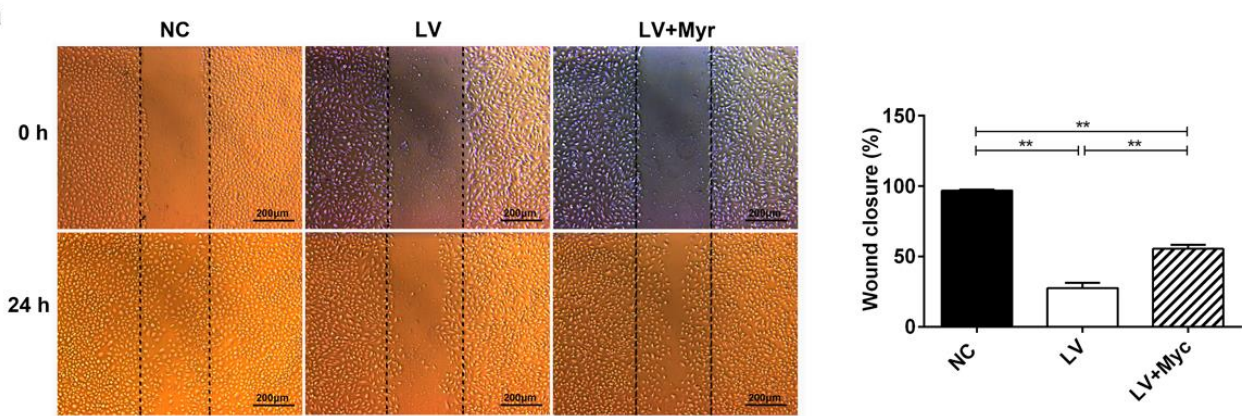

b
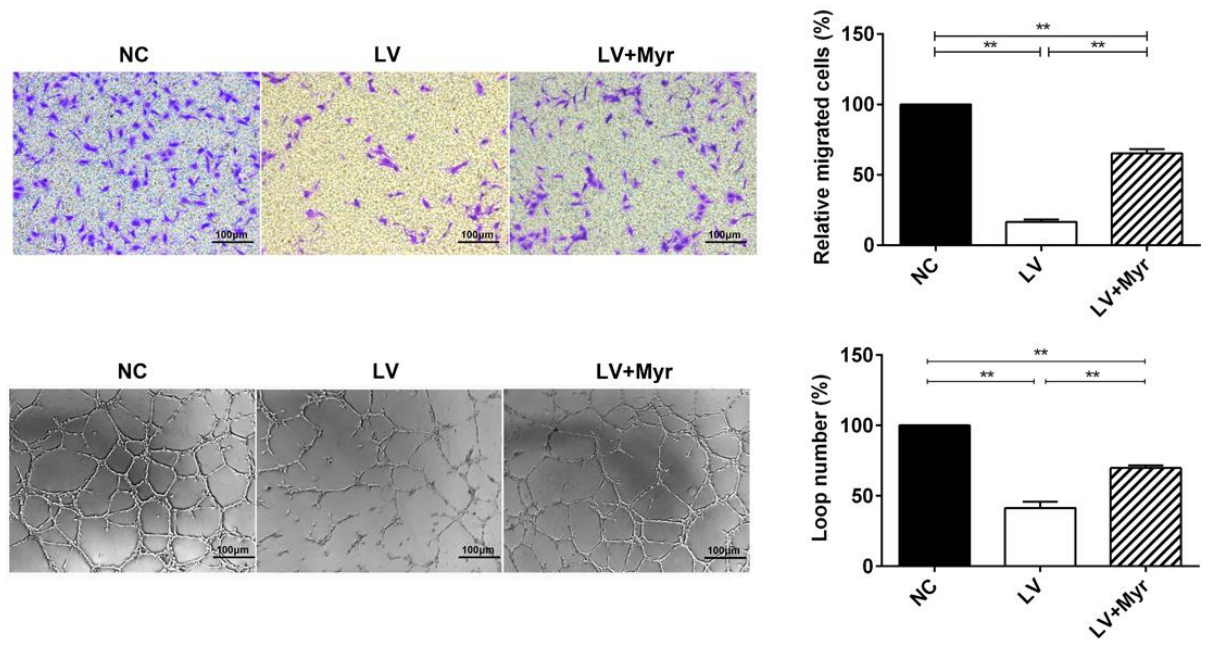

d
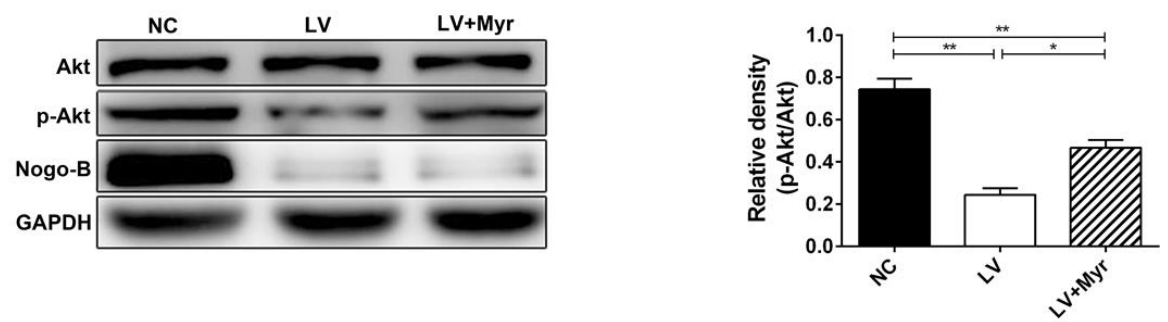

Fig. 5. Myriocin partially rescued the impairment of angiogenesis and Akt activation in Nogo-B knock down HRMECs. HRMECs were infected by lentivirus-siNogoB (LV group) and lentivirus negtive control (NC group). And cells were exposed to D-glucose (25 mM) after treatment with $100 \mathrm{nmol} / \mathrm{L}$ myriocin (Myr) or vehicle for 3 hours. The migration rate of HRMECs was determined in scratch wound assay (a) and transwell assay (b). The tube formation ability was assesed by plating the cells on matrigel (c). The expression of Akt and p-Akt was detected by western blot (d). $n=3$ in each group. Quantitative assessment of the data was presented in the right. Data were shown as mean $\pm \mathrm{SEM},{ }^{* *}, \mathrm{P}<0.01$.

in HRMECs infected by lentivirus. Similarly, 48 hour exposure to high D-glucose implied a considerable release of Nogo-B in the supernant of NC group and a relative paucity of Nogo-B in LV group (Fig. 6c). Besides, after medium replaced by fresh cell culture medium with normal D-glucose for additional 24 hours, the release of Nogo-B still persisted (Fig. 6d).

\section{Nogo-B promoted angiogenesis through PI3K/Akt activation in co-culture system}

Futhermore, we established co-culture system as described below. First, we cultured HRMECs infected by lentivirus on transwell inserts (NC and LV group) with high D-glucose treatment for 48 hours, then replaced with fresh complete growth medium with normal D-glucose for further culture, and seeded normal HRMECs to the bottom wells of chamber,

\section{KARGER}


a

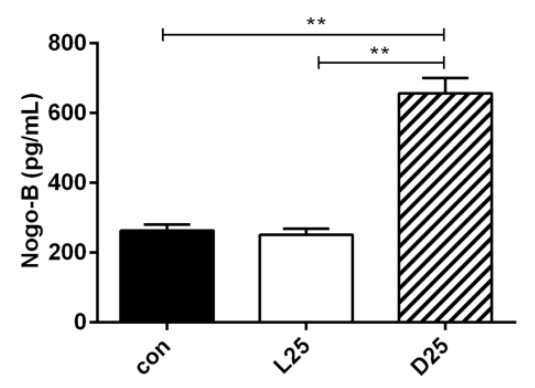

C

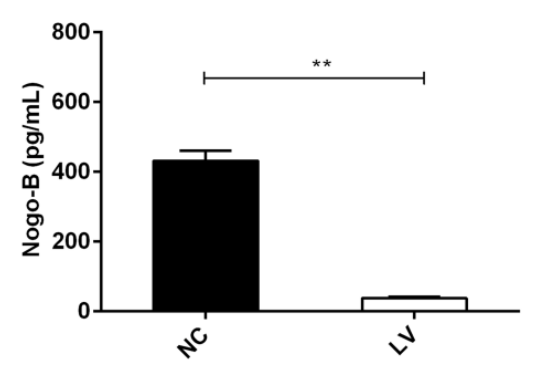

b

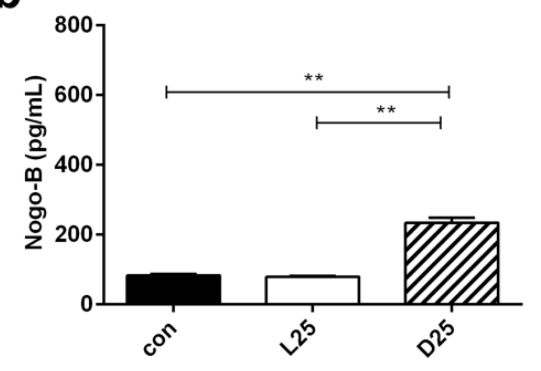

d

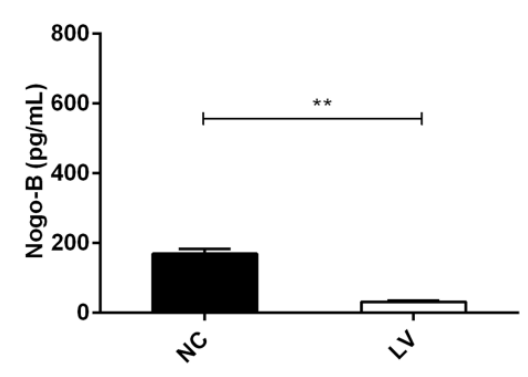

Fig. 6. High glucose treatment promoted the secretion of Nogo-B and presented as a "long-term memory". (a) ELISA analysis of Nogo-B in the supernant of HRMECs exposed to different glucose for 48 hours, $n=6$ in each group, (b) After treatment of different glucose for 48 hours, the supernant was discarded and replaced by fresh cell culture medium with normal glucose for further 24 hours, Elisa results of the supernants were presented as a histogram, $n=6$ in each group. (c) ELISA analysis of Nogo-B in the supernant of lentivirus infected HRMECs exposed to D-glucose (25mM) for 48 hours, $n=6$ in each group. (d) After treatment of Dglucose for 48 hours, the supernant was discarded and replaced by fresh cell culture medium with normal glucose for further 24 hours, Elisa results of the supernants were presented as a histogram, $n=6$ in each group. ${ }^{* *}, \mathrm{P}<0.001$.

the lower cells under LV group (LV-f group) showed blunted ability of migration and tube formation compared with the downside cells under NC group (NC-f group). However, the proliferation rate was not affected (Fig. 7a-g). Besides, PI3k p85 and Akt was activated in NC-f group compared with LV-f group (Fig. 7h, i).

\section{Discussion}

PDR, the stage of abnormal newly formed blood vessels grow from the retina, results in subsequntely tractional retinal detachment and hemorrhage, is the major concerning problem of DR $[32,33]$. Although the underlying mechanisms of aberrant angiogenesis in PDR is less than completely understood, lines of evidence shows that hyperglycemia is the most influential factor that contributes to the angiogenesis [34, 35]. Nogo-B, one of the evolutionary conservative proteins of endoplasmic reticulum that primarily play a role in promoting membrane curvature and tubules maintenance, was reported as a regulator of vascular remodeling and blood pressure, suggesting its role in vasvluar function $[21,22,36$, 37]. However, the role and function of Nogo-B in angiogenesis, especially in PDR have not been established yet. Therefore, we initially evaluated the presence of Nogo-B in the human PDR tissues and HRMECs in vitro. Strikingly, Nogo-B was enriched in both the vitreous and fibrovascular tissue samples of PDR patients. And stimulating of HRMECs with high glucose

\section{KARGER}


b

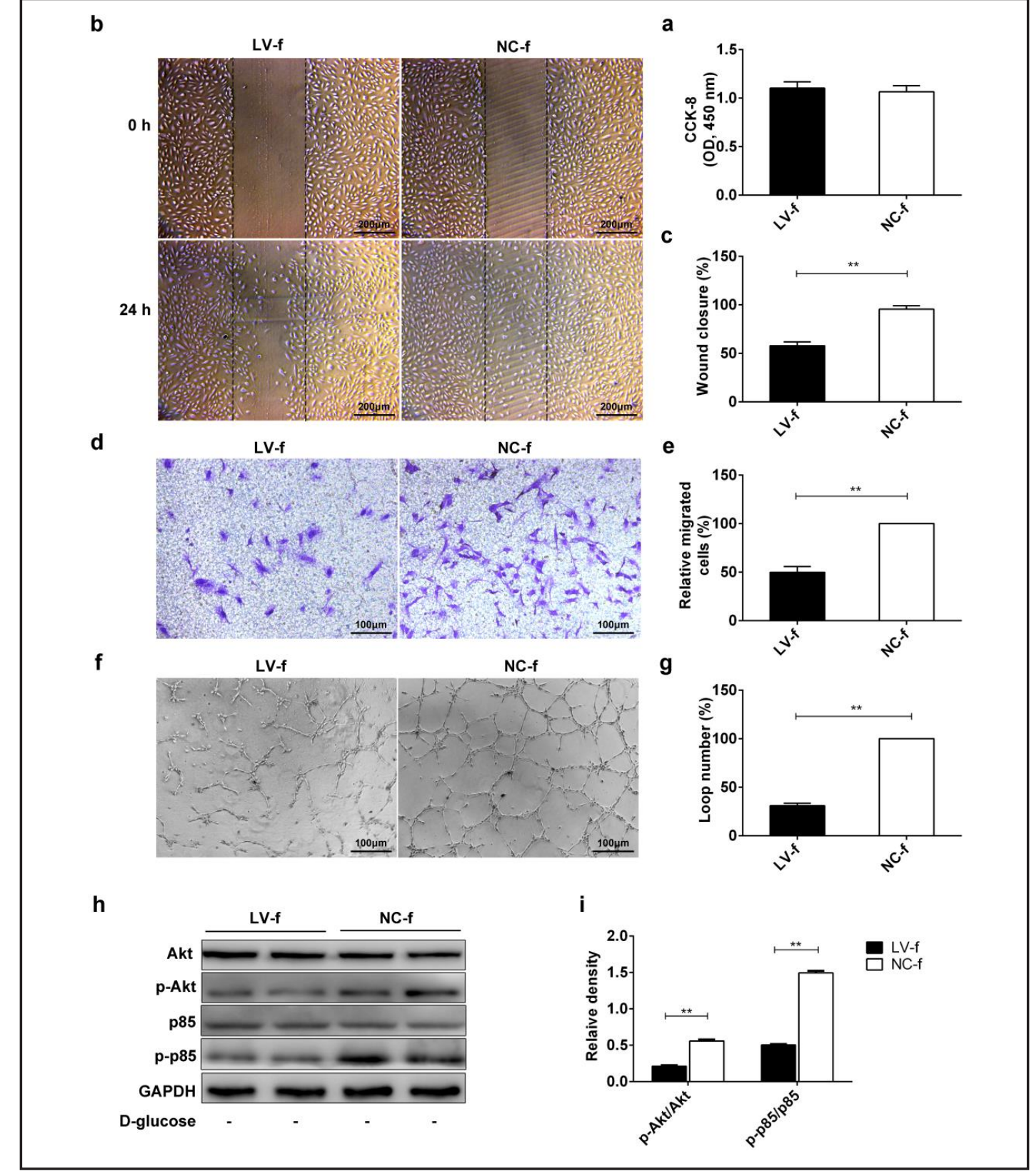

Fig. 7. Nogo-B promoted angiogenesis through PI3K/Akt activation in co-culture system. We established coculture system as described in methods, after fresh cell culture medium with normal glucose replacement, the lower cells under HRMECs infected by lentivrus were symboled as LV-f group and NC-f group. The cell viability was quantified and presented as a histogram by CCK-8 assay after treatment, $n=3$ in each group (a). The migration rate was determined in scratch wound assay, $n=3$ in each group (b, c) and transwell assay, $n=3$ in each group $(d, e)$. The tube formation ability was assesed by plating the cells on matrigel, $n=3$ in each group (f, g). Activation of PI3K p85 and Akt was detected using western blot (h, i). Data were shown as mean $\pm \mathrm{SEM}, * *, \mathrm{P}<0.01$ by student's t-test.

triggered the upregulation of Nogo-B. Therefore, we hypothesized that Nogo-B could likely be associated with angiogenesis in PDR.

To get insights into the participation of Nogo-B in angiogenesis of PDR, we use high glucose stimulation of HRMECs, an established cell culture model $[5,38]$. The cell proliferation assay, cell migration assay and tube formation assay are mature methods to imitate three

\section{KARGER}




\section{Cellular Physiology Cell Physiol Biochem 2017;43:1742-1754 \\ \begin{tabular}{l|l|l} 
DOI: 10.1159/000484061 & $\begin{array}{l}\text { O 2017 The Author(s). Published by S. Karger AG, Basel } \\
\text { www.karger.com/cpb }\end{array}$
\end{tabular} \\ Zhang et al.: Nogo-B Promotes Angiogenesis Via VEGF/PI3K/Akt Pathway}

critical steps of angiogenesis process. High glucose stimilation of HRMECs, which is the major target of hyperglycemia for its location of inner wall in blood vessel [39], resulted in considerable promotion of proliferation, migration and tube formation in HRMECs. And high glucose-induced angiogenesis was partialy reversed through downregulation of Nogo-B. The results suggested a novel aspect for the role of Nogo-B in high glucose condition for HRMECs as a positive regulator of angiogenesis, which was consistant with the previous reports demonstrated that Nogo-B promoted migration of HUVECs [21].

The underlying molecular mechanism of diabetic retinopathy is complex and remains largely elusive, which includes PI3K/Akt, p38 MAP kinase, calcium/calmodulin dependent protein kinase a, NOTCH/Wnt signalling [40-42]. Among them, an active VEGF/PI3K/Akt pathway has been dominated linked to pathological angiogenesis in PDR [28, 43, 44]. To analysis the molecular mechanisms and mediators of Nogo-B in regulation of angiogenesis, we examined VEGF secretion, as well as PI3K and Akt activation in lentivirus infected HRMECs. Our results showed that HRMECs exposed in high glucose elicited substantially activation of PI3K and Akt, and stimulated VEGF secretion, which was in consistant with accumulating previous reports $[27,28,43]$. In addition, downregulation of Nogo-B considerably blocked high glucose induced VEGF secretion, PI3K and Akt activation in HRMECs, indicating that Nogo-B may exert its angiogenesis actions through VEGF/PI3K/Akt pathway. Recently Nogo-B was reported to inhibit the sphingolipid biosynthesis through weakening the activity of serine palmitoyltransferase, the rate-limiting enzyme of the pathway [22]. Ceramide, a central metabolite of this pathway, was significantly upregulated in the absense of Nogo-B and has been shown to induce an inhibition of Akt activation [22,31, 45]. Our results showed that myriocin, an inhibitor of sphingolipid biosynthesis, partially rescued the impairment of angiogenesis and Akt activation in Nogo-B knock down HRMECs. However, the underlying mechanisms remained largely speculative and we will investigate further in our future study.

Considering the cell surface location of Nogo-B in endothelial cells and the extracellular domains exposed and released upon injury [21], we examined whether Nogo-B could be released to cell culture medium upon high glucose stimulation. Surprisingly, the relatively detectable of Nogo-B after exposure to high glucose was found in the present study. Besides, after a subsequent 24 hours of normal glucose culture, it was still apparently detectable in the cluture medium. To verify the role of Nogo-B in this phenomenon, we used co-cluture system with the upper chamber cultured with lentivrus infected HRMECs and normal HRMECs in the lower wells afterward, and added high glucose to the inserts. We found the secretion of Nogo-B persited after replacement of fresh medium for 24 hours, and the elevated Nogo-B in supernants could still promote angiogenesis of normal HRMECs in the downside chamber. These data indicated that HRMECs affected by high glucose could communicate with its neighboring normal cells through Nogo-B, which may contribute to the signalling cascade amplification of hyperglycemia in DR.

\section{Conclusion}

We reported that Nogo-B was highly enriched in ocular tissues of PDR patients and in HRMECs exposed to high glucose, and Nogo-B could be secreted upon high glucose exposure and acted as a positive regulator of angiogenesis in HRMECs partially through VEGF/PI3K/ Akt pathway, and the cell to cell communication between impaired HRMECs and neighboring normal cells could be ascribed to "the high glucose-induced elevation of Nogo-B memory". Therefore, elucidation of novel ways to downregulate the retinal level of Nogo-B or prevent its release can be a feasible therapeutic strategy for treating angiogenesis of PDR.

\section{Acknowledgements}

This work was supported by General Program of Shanghai Municipal Health and Family Planning Commission (201440522) and National Natural Science Foundation (81300777). 


\section{Cellular Physiology Cell Physiol Biochem 2017;43:1742-1754

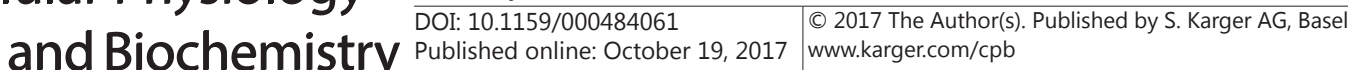 \\ Zhang et al.: Nogo-B Promotes Angiogenesis Via VEGF/PI3K/Akt Pathway}

\section{Disclosure Statement}

The authors declare that they have no Disclosure Statement.

\section{References}

1 Forbes JM, Cooper ME: Mechanisms of diabetic complications. Physiol Rev 2013;93:137-188.

-2 Zimmet P, Alberti KG, Magliano DJ, Bennett PH: Diabetes mellitus statistics on prevalence and mortality: facts and fallacies. Nat Rev Endocrinol 2016;12:616-622.

- 3 Liew G, Wong VW, Ho IV: Mini Review: Changes in the Incidence of and Progression to Proliferative and Sight-Threatening Diabetic Retinopathy Over the Last 30 Years. Ophthalmic Epidemiol 2017;24:73-80.

4 Roy S, Kern TS, Song B, Stuebe C: Mechanistic Insights into Pathological Changes in the Diabetic Retina: Implications for Targeting Diabetic Retinopathy. Am J Pathol 2017;187:9-19.

5 Stitt AW, Curtis TM, Chen M, Medina RJ, McKay GJ, Jenkins A, Gardiner TA, Lyons TJ, Hammes HP, Simo $\mathrm{R}$, Lois N: The progress in understanding and treatment of diabetic retinopathy. Prog Retin Eye Res 2016;51:156-186. Cheung N, Mitchell P, Wong TY: Diabetic retinopathy. Lancet 2010;376:124-136.

Klein R, Klein BE, Moss SE, Davis MD, DeMets DL: The Wisconsin Epidemiologic Study of Diabetic Retinopathy. X. Four-year incidence and progression of diabetic retinopathy when age at diagnosis is 30 years or more. Arch Ophthalmol 1989;107:244-249.

8 Klein R, Knudtson MD, Lee KE, Gangnon R, Klein BE: The Wisconsin Epidemiologic Study of Diabetic Retinopathy: XXII the twenty-five-year progression of retinopathy in persons with type 1 diabetes. Ophthalmology 2008;115:1859-1868.

$\rightarrow 9$ Scanlon PH, Stratton IM, Histed M, Chave SJ, Aldington SJ: The influence of background diabetic retinopathy in the second eye on rates of progression of diabetic retinopathy between 2005 and 2010. Acta Ophthalmol 2013;91:e335-339.

10 Simo R, Hernandez C: Advances in the medical treatment of diabetic retinopathy. Diabetes Care 2009;32:1556-1562.

11 Kusminski CM, Bickel PE, Scherer PE: Targeting adipose tissue in the treatment of obesity-associated diabetes. Nat Rev Drug Discov 2016;15:639-660.

12 Wei J, Jiang H, Gao H, Wang G: Blocking Mammalian Target of Rapamycin (mTOR) Attenuates HIF-1alpha Pathways Engaged-Vascular Endothelial Growth Factor (VEGF) in Diabetic Retinopathy. Cell Physiol Biochem 2016;40:1570-1577.

$>13$ Liu B, Li CP, Wang WQ Song SG, Liu XM: Lignans Extracted from Eucommia Ulmoides Oliv. Protects Against AGEs-Induced Retinal Endothelial Cell Injury. Cell Physiol Biochem 2016;39:2044-2054.

14 Ishimoto Y, Hirota-Takahata Y, Kurosawa E, Chiba J, Iwadate Y, Onozawa Y, Hasegawa T, Tamura A, Tanaka M, Kobayashi H: A Novel Natural Product-Derived Compound, Vestaine A1, Exerts both Pro-Angiogenic and Anti-Permeability Activity via a Different Pathway from VEGF. Cell Physiol Biochem 2016;39:1905-1918.

15 Mohamed Q, Gillies MC, Wong TY: Management of diabetic retinopathy: a systematic review. JAMA 2007;298:902-916.

-16 Oertle T, Schwab ME: Nogo and its paRTNers. Trends Cell Biology 2003;13:187-194.

17 Teng FY, Tang BL: Cell autonomous function of Nogo and reticulons: The emerging story at the endoplasmic reticulum. J Cell Physiol 2008;216:303-308.

18 GrandPre T, Nakamura F, Vartanian T, Strittmatter SM: Identification of the Nogo inhibitor of axon regeneration as a Reticulon protein. Nature 2000;403:439-444.

$>19$ Chen MS, Huber AB, van der Haar ME, Frank M, Schnell L, Spillmann AA, Christ F, Schwab ME: Nogo-A is a myelin-associated neurite outgrowth inhibitor and an antigen for monoclonal antibody IN-1. Nature 2000;403:434-439.

20 Kimura T, Endo S, Inui M, Saitoh S, Miyake K, Takai T: Endoplasmic Protein Nogo-B (RTN4-B) Interacts with GRAMD4 and Regulates TLR9-Mediated Innate Immune Responses. J Immunol 2015;194:5426-5436.

21 Acevedo L, Yu J, Erdjument-Bromage H, Miao RQ, Kim JE, Fulton D, Tempst P, Strittmatter SM, Sessa WC: A new role for Nogo as a regulator of vascular remodeling. Nat Med 2004;10:382-388.

$\checkmark 22$ Cantalupo A, Zhang Y, Kothiya M, Galvani S, Obinata H, Bucci M, Giordano FJ, Jiang XC, Hla T, Di Lorenzo A: Nogo-B regulates endothelial sphingolipid homeostasis to control vascular function and blood pressure. Nat Med 2015;21:1028-1037.

-23 Zhang Y, Huang Y, Cantalupo A, Azevedo PS, Siragusa M, Bielawski J, Giordano FJ, Di Lorenzo A: Endothelial 


\section{Cellular Physiology Cell Physiol Biochem 2017;43:1742-1754 \begin{tabular}{ll|l} 
and Biochemistry Published online: October 19, 2017 & $\begin{array}{l}\text { (c) } 2017 \text { The Author(s). Published by S. Karger AG, Basel } \\
\text { www.karger.com/cpb }\end{array}$ \\
\hline
\end{tabular}

Nogo-B regulates sphingolipid biosynthesis to promote pathological cardiac hypertrophy during chronic pressure overload. JCI Insight 2016. DOI: 10.1172/jci.insight.85484.

24 Wen M, Men R, Yang Z, Dan X, Wu W, Liu X, Yang L: The Value of Circulating Nogo-B for Evaluating Hepatic Functional Reserve in Patients with Cirrhosis. Dis Markers 2015;2015:419124. DOI: $10.1155 / 2015 / 419124$.

25 Potente M, Gerhardt H, Carmeliet P: Basic and therapeutic aspects of angiogenesis. Cell 2011;146:873-887.

26 Adams RH, Alitalo K: Molecular regulation of angiogenesis and lymphangiogenesis. Nat Rev Mol Cell Biol 2007;8:464-478.

-27 Yuan L, Hu J, Luo Y, Liu Q Li T, Parish CR, Freeman C, Zhu X, Ma W, Hu X, Yu H, Tang S: Upregulation of heparanase in high-glucose-treated endothelial cells promotes endothelial cell migration and proliferation and correlates with Akt and extracellular-signal-regulated kinase phosphorylation. Mol Vis 2012;18:16841695.

28 Zdychova J, Komers R: Emerging role of Akt kinase/protein kinase B signaling in pathophysiology of diabetes and its complications. Physiol Res 2005;54:1-16.

-29 Kureishi Y, Luo Z, Shiojima I, Bialik A, Fulton D, Lefer DJ, Sessa WC, Walsh K: The HMG-CoA reductase inhibitor simvastatin activates the protein kinase Akt and promotes angiogenesis in normocholesterolemic animals. Nat Med 2000;6:1004-1010.

30 Ackah E, Yu J, Zoellner S, Iwakiri Y, Skurk C, Shibata R, Ouchi N, Easton RM, Galasso G, Birnbaum MJ, Walsh $\mathrm{K}$, Sessa WC: Akt1/protein kinase Balpha is critical for ischemic and VEGF-mediated angiogenesis. J Clin Invest 2005;115:2119-2127.

-31 Hage Hassan R, Pacheco de Sousa AC, Mahfouz R, Hainault I, Blachnio-Zabielska A, Bourron O, Koskas F, Gorski J, Ferre P, Foufelle F, Hajduch E: Sustained Action of Ceramide on the Insulin Signaling Pathway in Muscle Cells: IMPLICATION OF THE DOUBLE-STRANDED RNA-ACTIVATED PROTEIN KINASE. J Biol Chem 2016;291:3019-3029.

-32 Crawford TN, Alfaro DV, 3rd, Kerrison JB, Jablon EP: Diabetic retinopathy and angiogenesis. Curr Diabetes Rev 2009;5:8-13.

-33 Brownlee M: Biochemistry and molecular cell biology of diabetic complications. Nature 2001;414:813-820.

34 Nyengaard JR, Ido Y, Kilo C, Williamson JR: Interactions between hyperglycemia and hypoxia: implications for diabetic retinopathy. Diabetes 2004;53:2931-2938.

-35 Ye EA, Steinle JJ: miR-146a Attenuates Inflammatory Pathways Mediated by TLR4/NF-kappaB and TNFalpha to Protect Primary Human Retinal Microvascular Endothelial Cells Grown in High Glucose. Mediators Inflamm 2016;2016:3958453. DOI: 10.1155/2016/3958453.

-36 Jozsef L, Tashiro K, Kuo A, Park EJ, Skoura A, Albinsson S, Rivera-Molina F, Harrison KD, Iwakiri Y, Toomre D, Sessa WC: Reticulon 4 is necessary for endoplasmic reticulum tubulation, STIM1-Orai1 coupling, and storeoperated calcium entry. J Biol Chem 2014;289:9380-9395.

-37 Zurek N, Sparks L, Voeltz G: Reticulon short hairpin transmembrane domains are used to shape ER tubules. Traffic 2011;12:28-41.

38 Yokouchi H, Eto K, Nishimura W, Takeda N, Kaburagi Y, Yamamoto S, Yasuda K: Angiopoietin-like protein 4 (ANGPTL4) is induced by high glucose in retinal pigment epithelial cells and exhibits potent angiogenic activity on retinal endothelial cells. Acta Ophthalmol 2013;91:e289-297.

39 Tian J, Ma H, Luo Y, Hu A, Lin S, Li T, Guo K, Li J, Cai M, Tang S: The influence of high glucose on the Cip/Kip family expression profiles in HRECs. J Mol Histol 2013;44:705-713.

40 Carmeliet P, Jain RK: Molecular mechanisms and clinical applications of angiogenesis. Nature 2011;473:298-307.

41 Veluthakal R, Kumar B, Mohammad G, Kowluru A, Kowluru RA: Tiam1-Rac1 Axis Promotes Activation of p38 MAP Kinase in the Development of Diabetic Retinopathy: Evidence for a Requisite Role for Protein Palmitoylation. Cell Physiol Biochem 2015;36:208-220.

42 Li J, Wang P, Ying J, Chen Z, Yu S: Curcumin Attenuates Retinal Vascular Leakage by Inhibiting Calcium/ Calmodulin-Dependent Protein Kinase II Activity in Streptozotocin-Induced Diabetes. Cell Physiol Biochem 2016;39:1196-1208.

43 Shiojima I, Walsh K: Role of Akt signaling in vascular homeostasis and angiogenesis. Circ Res 2002;90:1243-1250.

44 Gliki G, Wheeler-Jones C, Zachary I: Vascular endothelial growth factor induces protein kinase C (PKC)dependent Akt/PKB activation and phosphatidylinositol 3'-kinase-mediates PKC delta phosphorylation: role of PKC in angiogenesis. Cell Biol Int 2002;26:751-759.

45 Rahman A, Thayyullathil F, Pallichankandy S, Galadari S: Hydrogen peroxide/ceramide/Akt signaling axis play a critical role in the antileukemic potential of sanguinarine. Free Radic Biol Med 2016;96:273-289. 